\title{
Profiling bacterial communities by MinION sequencing of ribosomal operons
}

\author{
Lee J. Kerkhof ${ }^{1 *} \mathbb{D}$, Kevin P. Dillon², Max M. Häggblom ${ }^{3}$ and Lora R. McGuinness ${ }^{1}$
}

\begin{abstract}
Background: An approach utilizing the long-read capability of the Oxford Nanopore MinION to rapidly sequence bacterial ribosomal operons of complex natural communities was developed. Microbial fingerprinting employs domain-specific forward primers (16S rRNA subunit), reverse primers (23S rRNA subunit), and a high-fidelity Taq polymerase with proofreading capabilities. Amplicons contained both ribosomal subunits for broad-based phylogenetic assignment ( 3900 bp of sequence), plus the intergenic spacer (ITS) region ( 300 bp) for potential strain-specific identification.

Results: To test the approach, bacterial rRNA operons ( 4200 bp) were amplified from six DNA samples employing a mixture of farm soil and bioreactor DNA in known concentrations. Each DNA sample mixture was barcoded, sequenced in quadruplicate $(n=24)$, on two separate 6 -h runs using the MinION system (R7.3 flow cell; MAP005 and 006 chemistry). From nearly 90,000 MinION reads, roughly 33,000 forward and reverse sequences were obtained. This yielded over 10,000 2D sequences which were analyzed using a simplified data analysis pipeline based on NCBI Blast and assembly with Geneious software. The method could detect over 1000 operational taxonomic units in the sample sets in a quantitative manner. Global sequence coverage for the various rRNA operons ranged from 1 to 1951x. An iterative assembly scheme was developed to reconstruct those rRNA operons with $>35 x$ coverage from a set of 30 operational taxonomic units (OTUs) among the Proteobacteria, Actinobacteria, Acidobacteria, Firmicutes, and Gemmatimonadetes. Phylogenetic analysis of the $16 \mathrm{~S}$ rRNA and $23 \mathrm{~S}$ rRNA genes from each operon demonstrated similar tree topologies with species/strain-level resolution.
\end{abstract}

Conclusions: This sequencing method represents a cost-effective way to profile microbial communities. Because the MinION is small, portable, and runs on a laptop, the possibility of microbiota characterization in the field or on robotic platforms becomes realistic.

Keywords: Microbiota, Ribosomal operon, MinION, Species/strain-level resolution

\section{Background}

Molecular biological approaches for the genetic analysis of environmental samples have become the most widely accepted way to characterize microbial communities. Initially, a clone and sequence scheme was largely used to characterize $5 \mathrm{~S}$ or $16 \mathrm{~S}$ rRNA genes [1,2]. Later, direct profiling methods such as denaturing gradient gel electrophoresis (DGGE), terminal restriction fragment length polymorphism (TRFLP), or single strand conformation polymorphism (SSCP) analysis were employed to characterize complex communities [3-5].

\footnotetext{
*Correspondence: Ikerkhof@rutgers.edu

${ }^{1}$ Department of Marine and Coastal Sciences, Rutgers University, 71 Dudley

Rd, New Brunswick, NJ 08901-8521, USA

Full list of author information is available at the end of the article
}

More recently, 16S rRNA gene sequence data is collected using a suite of "next generation" sequencing platforms (e.g., 454, Illumina, Ion Torrent) [6-9]. Although a large amount of data can be obtained in this manner, these recent approaches rely on expensive machines, bioinformatics training, and specialized computing facilities. In order to analyze the sequence data, a working knowledge of UNIX commands and Python scripts seems essential. Despite the computer software being freely available (e.g., QIIME and Mothur), the programs require the use of command lines and training in the proper UNIX syntax in order to function properly. Often the installation scripts and software dependencies become outdated quickly, and it is not always straightforward to install and/or operate. Furthermore, a server 
is generally needed to perform the analyses. Each of these requirements can place a significant monetary burden and a steep learning curve onto laboratories hoping to characterize bacterial communities. Finally, most second-generation sequencing platforms provide relatively short read lengths (200-400 bp) which limit the phylogenetic depth that can be achieved (with the exception of the PacBio system).

As an alternative approach, we tested if the portable DNA sequencer (MinION) from Oxford Nanopore Technologies (ONT) could be used to profile the microbiota using tools that can be purchased for a low cost and data analysis methods that are readily available to many laboratories. The MinION is a third-generation platform for direct sequencing of individual strands of DNA translocating nanoscale pores in a semiconductor membrane $[10,11]$. A major advantage of the MinION is that it currently costs $\sim \$ 1000$, connects to a laptop, collects/analyzes data in real time, and does not require specialized computer equipment or training for data analysis. For MinION sequencing, each DNA molecule has an adaptor ligated to one end, which interacts with a docking protein and binds to a nanopore. This docking protein regulates the speed by which the DNA traverses the membrane. The other end of the DNA fragment is ligated to a hairpin structure, which allows for the complementary strand to be sequenced as it flows through the pore. The DNA sequence is determined from 5-bp segments (k-mers) by measuring the change in electrical conductivity across the membrane as the DNA strand flows along the nanopore channel using hidden Markov models and Metrichor base calling software which is available to MinION users on the web. This approach generates 2D (double stranded; template plus complement) reads for single DNA molecules possessing both adaptor and hairpin, while 1D (single stranded; template or complement) reads are generated for DNA molecules possessing only the adaptor. (Those DNA molecules without adaptor or hairpin are removed during library preparation and are not detected in the analysis.)

For this study, we tested whether the MinION could be used to rapidly sequence bacterial ribosomal operons from complex environmental samples. To validate the approach, we generated a mixture of complex genomic DNA from two different sources where a large number of unknown microorganisms exist rather than a simple mock community of a few model organisms. After rRNA operon sequencing, each individual read was assigned to an operational taxonomic unit (OTU) by screening against an NCBI 16S rRNA gene database. An rRNA consensus sequence was then reconstructed for a particular OTU using an iterative alignment approach with a commercially available DNA software program (Geneious; < \$900 per academic license) which can be run on Windows, Mac, or Linux operating systems. These efforts were designed to test if consensus building would yield data for environmental rRNA operons that are reproducible, quantitative, and similar to known rRNA genes within online databases.

In order to determine if the MinION can provide relative abundance data for the rRNA operons from environmental samples, genomic DNA from two different complex microbial communities were mixed in known concentrations (Rutgers farm soil and a NASA gray water bioreactor; [12]). These samples were chosen to represent environmental and engineered systems containing hundreds to thousands of different bacterial OTUs at varying concentrations. Six samples were constructed using mixtures of farm soil and bioreactor DNA at known concentrations to allow for replication and to test for the ability to provide relative abundance information (Fig. 1). Microbial fingerprinting employed domain-specific forward primers (16S rRNA gene subunit), domain-specific reverse primers (23S rRNA gene subunit), and a high-fidelity Taq polymerase with proofreading capabilities. The MinION data was processed with a user-friendly analysis pipeline in Geneious 10 that mirrors QIIME including denoising, OTU calling, assembling, and phylogenetic analysis (Additional file 1: Figure S1). Over 1000 OTUs (unique matches to the NCBI 165 rRNA gene database) were detected in the environmental dataset. Once the individual operon sequences were grouped by OTU, the various sequencing errors (i.e., miscalls, insertions, deletions; Additional file 1: Figure S2) were eliminated by consensus building. This consensus building approach demonstrated that nearly complete rRNA operons could be reconstructed from the MinION environmental dataset, containing $16 \mathrm{~S}$ and $23 \mathrm{~S}$ rRNA genes from members of different bacterial phyla. Both ribosomal RNA subunits yielded near identical phylogenetic tree topologies. Furthermore, MinION rRNA operon profiling was shown to be quantitative and provide species/strain-level resolution. Therefore, the MinION is a cost-effective alternative for profiling the microbiota that is small and portable and can be used in the field or on autonomous platforms.

\section{Results}

Soil and bioreactor DNA were combined in known proportions to generate six sample communities to test the ability of the MinION to sequence nearly complete rRNA operons and determine if read numbers could measure the relative abundance of the different OTUs (Fig. 1). After purification of genomic DNA with Agencourt AMPure beads (Beckman Coulter; Brea, CA, USA), a 4.2-kb amplicon was created from the bacteria rRNA operons using 16S rRNA gene (27F) and 23S 


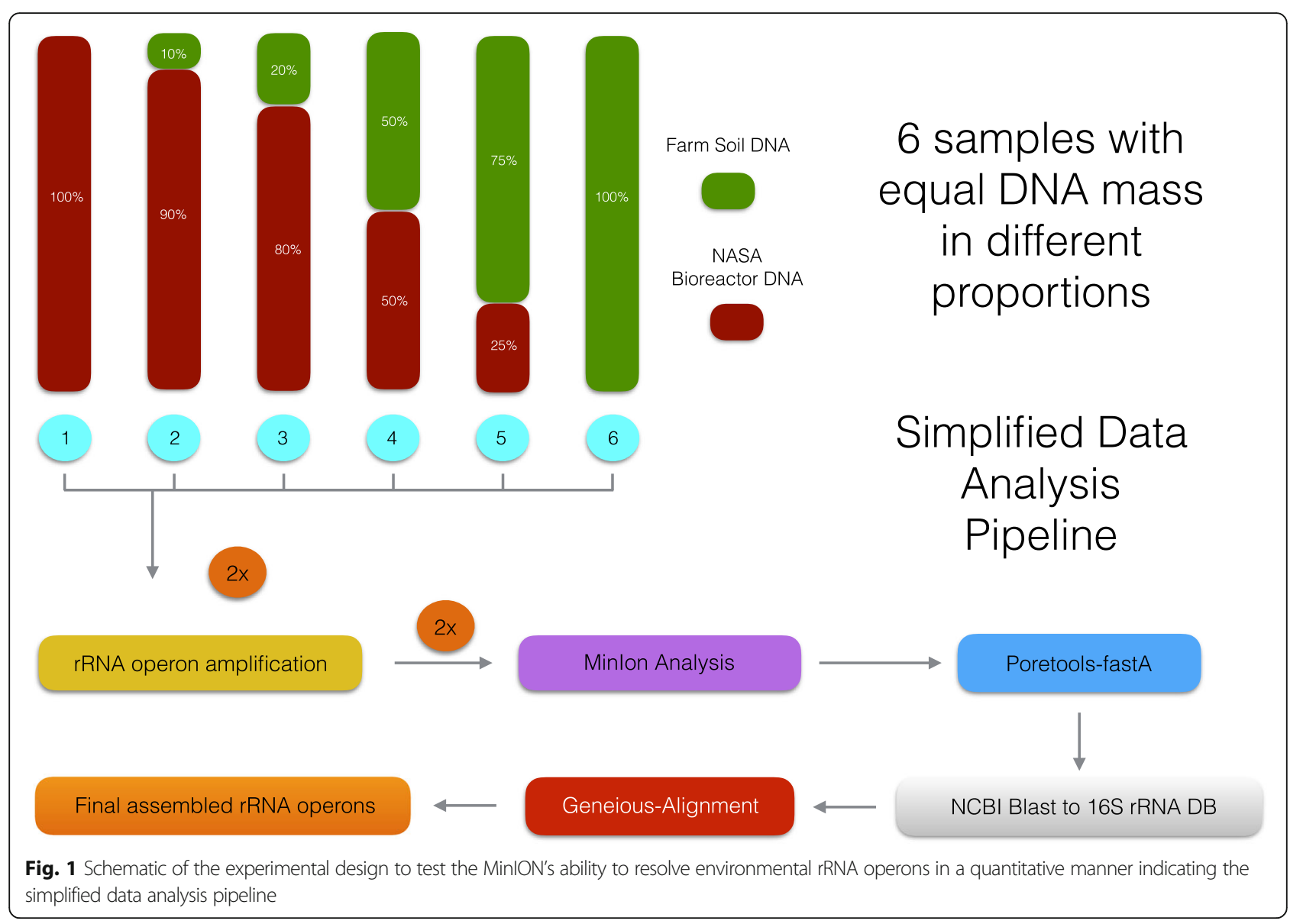

rRNA gene (2241R) primers (Additional file 1: Figure S3). The amplicons were barcoded using slight modifications of the Oxford Nanopore barcoding kit and R7.3 flow cells/ chemistry. This yielded 12 barcoded samples per flow cell. A second set of rRNA operon libraries was also created for biological replication (e.g., separate DNA extractions, amplification, barcoding, ligations) and sequenced on a second flow cell, yielding quadruplicate sample replication for this study. Overall, nearly 90,000 reads totaling over $350 \mathrm{Mbp}$ were obtained in two 6-h runs on the MinION. Most of the reads were 1D, representing sequences predominantly in the forward or reverse direction (Additional file 1: Table S1). However, roughly 33,000 reads containing both forward and reverse strands were recovered, yielding over 10,000 2D sequences generated by Metrichor. The MinION reads were analyzed via a simplified data analysis pipeline based on a local Discontinuous MegaBLAST search to a $16 \mathrm{~S}$ rRNA gene database (bacteria and archaea; Bioproject 33175) using Geneious 10.1.2. This BLAST approach could detect over 1000 OTUs within the global data set (Fig. 2a) with coverage ranging from 1 to $1951 x$ (Table 1). To ascertain if MinION yielded comparable proportions of identified OTUs with respect to the number of raw sequence reads as other next-generation sequencing platforms, rarefaction analysis was performed (Fig. 2b). Our results were similar to other published reports of microbial diversity using Illumina, pyrosequencing, and PacBio methods from mangroves, soils, dhole/dog feces, aquaculture ponds, the deep sea, waste water bioreactors, fruit fly gut, and cow rumen (Illumina methods-[13-16]; 454 methods- [6, 17-19]; PacBio[20]). However, MinION OTU yields for the bioreactor and soil DNA were lower than studies of the marine samples. Additionally, we screened the MinION reads using ARB/SINA and the Greengenes, RDP, and SILVA SSU databases. This re-analysis indicated that NCBI and SILVA databases provided the lowest percentage of unclassified OTUs (i.e., either defined as unclassified in the database or $<70 \%$ identity over 500 bp; Additional file 1: Figure S4).

Because the 2D sequencing error rate for MinION reads has been reported at $12 \%$ [21], we performed a sensitivity analysis to determine whether MinION reads with comparable errors could be accurately assigned to an OTU by Discontinuous MegaBLAST. Three 16S rRNA gene sequences from the NCBI database (Stenotrophomonas 


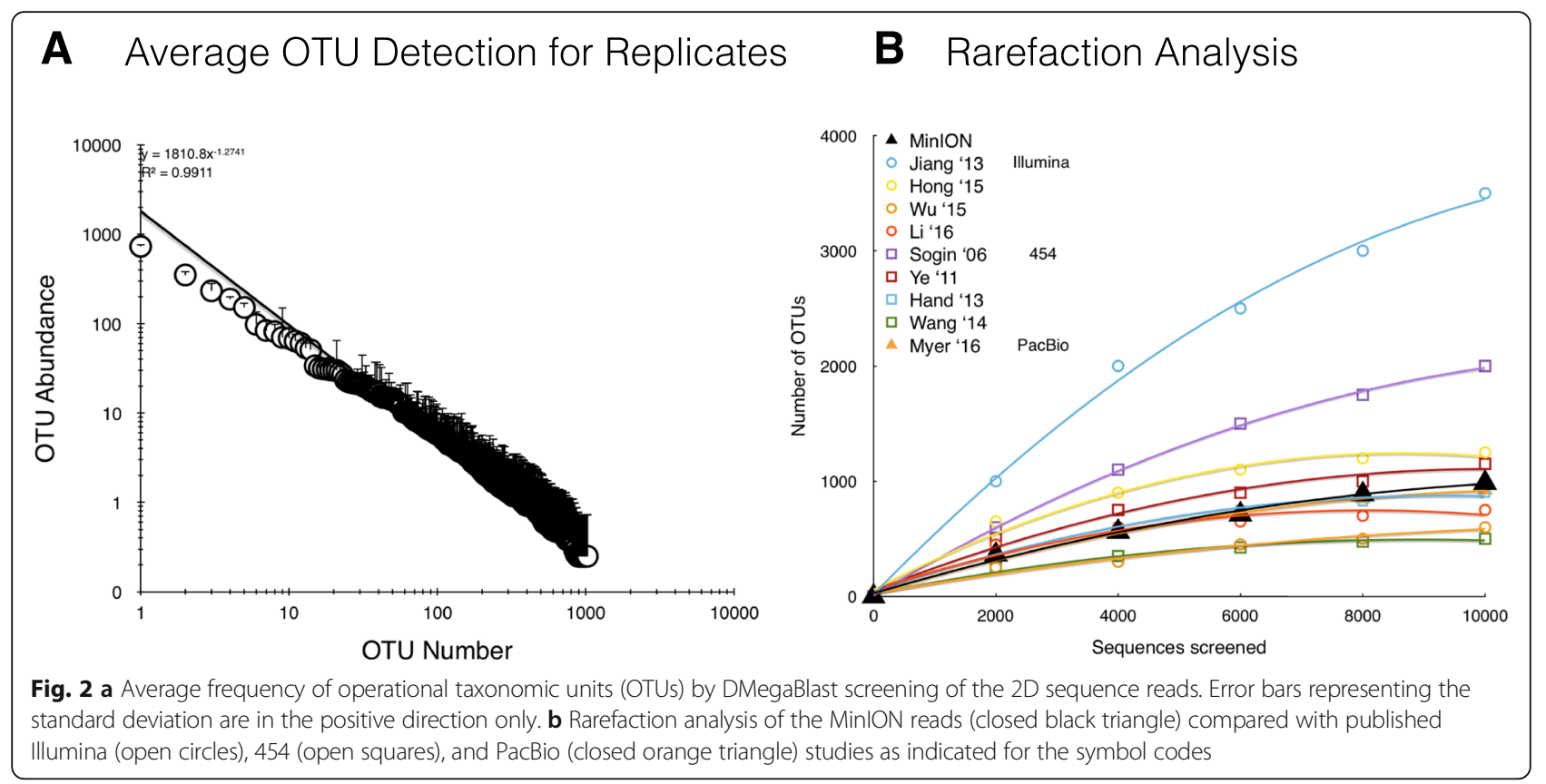

maltophilia, Comomonas nitrativorans, Comomonas denitrificans) had random errors and indels introduced along the entire length creating copies with similarities ranging from 79 to $100 \%$ (Additional file 1: Figure S5). All these test sequences were screened by Discontinuous MegaBLAST as described above and were assigned to the proper source OTU with the appropriate substitution rate (Additional file 1: Table S2).

Although equal masses of amplicons from the various mixtures were used for library construction, the different barcodes did not provide a uniform number of $2 \mathrm{D}$ operon sequences per barcode (Additional file 1: Figure S6). However, once normalized, the 2D data indicated highly reproducible diversity patterns for the quadruplicate samples with most of the variability in OTUs below 10 hits (Fig. 2a). Since the contribution of the soil and bioreactor genomic DNA to each sample was known, it was possible to test if the various MinION OTUs were represented in a linear manner in the MinION reads. The top four normalized OTUs from the soil and bioreactor DNA provided a linear, quantitative signal with $r^{2}$ values $\sim 0.9$ for the most abundant OTUs (Fig. 3). Those less abundant OTUs with more than three data points for the DNA mixtures were also linearly correlated with the proportion of input DNA $(n=104)$. However, the robustness of this correlation declines with the number of OTUs $<10$ within the sample set (Additional file 1: Figure S7).

In order to reconstruct those rRNA operons with $>35 \mathrm{x}$ coverage, an iterative assembly scheme using LastZ alignment [22] was employed (Fig. 4). The results yielded nearly intact rRNA operons from members of the Proteobacteria, Actinobacteria, Acidobacteria, Firmicutes, and
Gemmatimonadetes, containing most of the $16 \mathrm{~S}$ and $23 \mathrm{~S}$ rRNA subunits (examples in Additional file 1: Figure S8). The robustness and error rate of the consensus building scheme was tested by building consensus sequences from the four biological replicates. Here, three OTUs were identified from the bioreactor DNA end-member sample that had $30+$ reads in each of the four barcoded samples (Acidovorax wautersii, Comomonas nitrativorans, Stenotrophomonas rhizophilia). The $16 \mathrm{~S}$ rRNA genes from these biological replicates were independently aligned and used to build independent consensus sequences for each replicate as was done for the entire rRNA operon. All four replicate consensus sequences for the three OTUs were found to be identical (Additional file 1: Figure S9), indicating that our consensus building approach introduces minimal errors.

To test whether the reconstructed rRNA operons contained chimeric rRNA genes, phylogenetic analysis was performed separately on the $16 \mathrm{~S}$ and the $23 \mathrm{~S}$ rRNA genes using 1292 and 1767 unambiguously aligned positions, respectively. Unfortunately, the $23 \mathrm{~S}$ rRNA gene database is significantly smaller than the 16S rRNA gene database and UCHIME or similar software do not have a database that links the $16 \mathrm{~S}$ and $23 \mathrm{~S}$ rRNA genes. All MinION sequences using this method contained near identical phylogenetic tree topologies for both rRNA subunits as demonstrated for the Proteobacteria (Figs. 5 and 6; and the other bacterial phyla; Additional file 1: Figures S10-S13). Finally, the reconstructed $16 \mathrm{~S}$ rRNA genes were re-screened against the NCBI NR database to assess if the top BLAST hits from the initial $16 \mathrm{~S}$ rRNA 
Table 1 Discontinuous MegaBLAST results for the rRNA consensus operons

\begin{tabular}{|c|c|c|c|c|c|c|}
\hline NCBI top hit & Phylum & Bacterium & $x$ coverage & Size (bp) & $16 S \% I^{*}$ & Overlap* \\
\hline gi|270610434|gb|GU187030.1| & Acidobacteria & Acidobacteria bacterium IGE-003 & 91 & 4022 & $83.8 \pm 3.1$ & $1345 \pm 161$ \\
\hline gi|584432076|gb|KF840371.1| & Acidobacteria & Stenotrophobacter terrae & 59 & 4269 & $82.6 \pm 5.0$ & $1445 \pm 83$ \\
\hline gi|270610434|gb|GU187030.1|| & Acidobacteria & Acidobacteria bacterium IGE-003 *** & 56 & 4088 & $90.0 \pm 4.7$ & $622 \pm 251$ \\
\hline gi|270610434|gb|GU187030.1| & Acidobacteria & Acidobacteria bacterium IGE-003 *** & 37 & 3933 & $84.7 \pm 2.7$ & $1324 \pm 128$ \\
\hline gi|645321086|ref|NR_118138.1| & Actinobacteria & Gaiella occulata & 444 & 4006 & $86.6 \pm 2.6$ & $1483 \pm 69$ \\
\hline gi|283945692|gb|CP001854.1| & Actinobacteria & Conexibacter woesei & 161 & 4148 & $88.3 \pm 3.7$ & $1472 \pm 85$ \\
\hline gi|631251774|ref|NR_112972.1| & Actinobacteria & Aciditerrimonas ferrireducens & 98 & 3803 & $87.6 \pm 1.4$ & $1497 \pm 33$ \\
\hline gi|772393478|gb|KP174569.1 & Actinobacteria & lamia majanohamensis & 50 & 4257 & $87.2 \pm 1.6$ & $1509 \pm 41$ \\
\hline gi|566084828|ref|NR_108192.1| & Actinobacteria & Solirubrobacter ginsenosidimutans & 45 & 3655 & $86.1 \pm 3.5$ & $1446 \pm 88$ \\
\hline gi|343200946|ref|NR_041633.1| & Actinobacteria & Ilumatobacter fluminis & 41 & 4167 & $89.3 \pm 1.9$ & $1279 \pm 38$ \\
\hline gi|294768454|gb|GU905013.1| & Firmicutes & Enterococcus sp. CSL 7544-3 & 68 & 4167 & $96.7 \pm 2.1$ & $1491 \pm 52$ \\
\hline gi|219846897|ref|NR_026489.1| & Firmicutes & Vagococcus fluvialis & 46 & 4153 & $97.0 \pm 2.1$ & $1489 \pm 51$ \\
\hline gi|37961728|gb|AY234571.1| & Gemmatimonadetes & Bacterium Ellin5220 & 316 & 3611 & $84.0 \pm 2.7$ & $1039 \pm 6$ \\
\hline gi|68146509|emb|AJ867290.1| & Proteobacteria-alpha & Ochrobactrum anthropi & 61 & 4567 & $98.2 \pm 0.8$ & $1530 \pm 24$ \\
\hline gi|219878237|ref|NR_025376.1 & Proteobacteria-beta & Comomonas nitrativorans & 941 & 4623 & $94.4 \pm 1.0$ & $1496 \pm 40$ \\
\hline gi|111378460|gb|DQ836252.1| & Proteobacteria-beta & Comomonas denitrificans & 384 & 4585 & $96.3 \pm 1.4$ & $1501 \pm 43$ \\
\hline gi|698376506|gb|KM210263.1| & Proteobacteria-beta & Castellaniella sp. ADC-27 & 118 & 4652 & $95.2 \pm 1.5$ & $1502 \pm 51$ \\
\hline gi|589264544|emb|HG916765.1| & Proteobacteria-beta & Castellaniella defragrans & 88 & 4519 & $95.1 \pm 1.4$ & $1513 \pm 43$ \\
\hline gi|343201701|ref|NR_042427.1| & Proteobacteria-beta & Acidovorax caeni & 80 & 4432 & $95.4 \pm 0.8$ & $1521 \pm 30$ \\
\hline gi|937501645|gb|KR136349.1| & Proteobacteria-beta & Achromobacter xylosoxidans & 70 & 4093 & $97.6 \pm 0.2$ & $1521 \pm 16$ \\
\hline gi|219878311|ref|NR_025450.1| & Proteobacteria-beta & Bacterium Ellin6067 & 39 & 3967 & $85.5 \pm 0.4$ & $1551 \pm 10$ \\
\hline gi|410994849|gb|CР003920.1| & Proteobacteria-epsilon & Sulfuricurvum kujiense & 148 & 4453 & $89.1 \pm 3.7$ & $1499 \pm 45$ \\
\hline gi|54887524|emb|AJ786786.1| & Proteobacteria-gamma & Xanthomonas sp. R-20819 & 1951 & 4479 & $95.1 \pm 1.0$ & $1521 \pm 27$ \\
\hline gi|28170769|dbj|AB101447.1| & Proteobacteria-gamma & Xanthomonas axonopodis & 352 & 4471 & $94.7 \pm 1.0$ & $1531 \pm 27$ \\
\hline gi|828983113|gb|CP011657.1| & Proteobacteria-gamma & Citrobacter freundii CAV1741 & 244 & 4248 & $98.2 \pm 0.3$ & $1534 \pm 11$ \\
\hline gi|938169631|gb|CP012900.1| & Proteobacteria-gamma & Stenotrophomonas acidaminiphila & 161 & 4272 & $92.5 \pm 0.5$ & $1253 \pm 5$ \\
\hline gi|28170769|dbj|AB101447.1| & Proteobacteria-gamma & Xanthomonas axonopodis *** & 113 & 4367 & $94.2 \pm 1.2$ & $1457 \pm 30$ \\
\hline gi|927043620|gb|CP012554.1| & Proteobacteria-gamma & Citrobacter freundii P10159 & 80 & 4177 & $97.9 \pm 0.4$ & $1536 \pm 14$ \\
\hline gi|927043620|gb|CP012554.1| & Proteobacteria-gamma & Citrobacter freundii P10159*** & 55 & 4286 & $97.2 \pm 0.3$ & $1540 \pm 11$ \\
\hline gi|636559888|ref|NR_115948.1| & Proteobacteria-gamma & Lysobacter dokdonensis & 38 & 4361 & $93.7 \pm 0.5$ & $1545 \pm 16$ \\
\hline
\end{tabular}

${ }^{*}$ Averages \pm stdev from the top 100 BLAST hits from the NR database; *** $16 \mathrm{~S}$ rRNA genes with different top Discontinuous MegaBLAST hits using the $16 \mathrm{~S}$ rRNA gene and NR databases

database screen were also obtained from a much larger data set. In 26 out of 30 rRNA operons, the NR screen retrieved the identical top hit as the screen $16 \mathrm{~S}$ rRNA dataset. In the remaining four rRNA operons, the 16S rRNA top hit was within the top three hits in the NR dataset. To gain a sense of the similarity between the reconstructed $16 \mathrm{~S}$ rRNA genes and entries in the NR database, the average overlap and identity for the top 100 hits were calculated (Table 1). Most of the reconstructed rRNA operons (28 out of 30) retrieve nearly full-length BLAST hits from the NR database (> $1250 \mathrm{bp}$; most being $\sim 1500 \mathrm{bp}$ ). Half of the reconstructed rRNA operons had identities $>93 \%$ for their respective matches. The other half of the reconstructed rRNA operons were not well represented in the 16S rRNA gene or NR databases and had similarities $<93 \%$, as is often found when screening environmental samples.

\section{Discussion}

Direct amplification of 16S rRNA genes from genomic DNA has revolutionized our understanding of the complexity of microbial communities. However, most recent efforts devoted to 16S rRNA gene discovery have focused more on the volume of sequences rather than the length of the sequence obtained from the molecule. It is now common to use high-throughput sequencing methods (e.g., Illumina, Ion Torrent, Pyrosequencing) to 


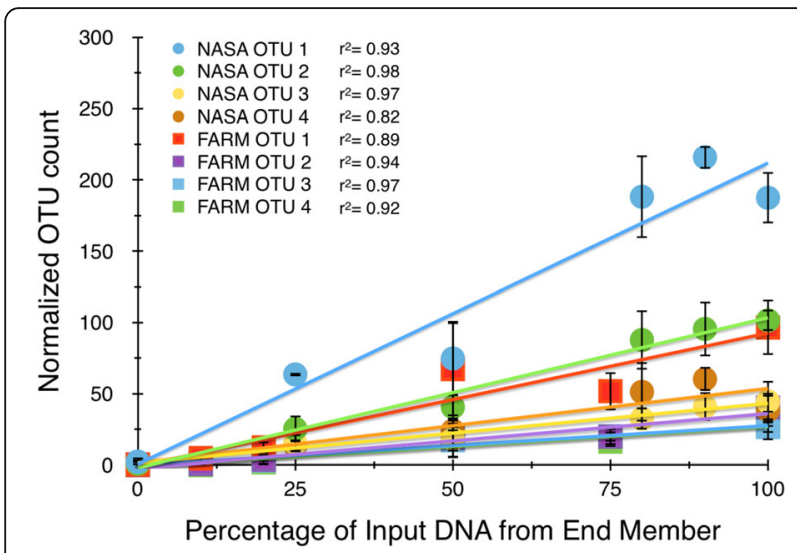

Fig. 3 Quantitative response of the four most abundant OTU reads (farm soil and bioreactor; $y$ axis) versus the percentage of input DNA from the end-member DNAs ( $x$ axis). Error bars represent the standard deviation

generate millions of short reads (often $<200-400 \mathrm{bp}$ ) and to report results at the phylum-order-family level. This approach inherently groups all members of a bacterial phyla-order-family together into a single unit and obscures species or strain-level dynamics that may be occurring in an environmental or experimental perturbation (e.g., light, temperature, nutrient addition). In this study, we tested a portable sequencing technology for the ability to distinguish bacterial species or strains in environmental samples. The Oxford MinION sequences single DNA molecules and enables very long reads to be obtained, compared to most secondgeneration sequencing approaches $(>10,000$ bp vs $200 \mathrm{bp}$ ). When applied to rRNA gene characterization, this approach can provide nearly full-length rRNA operon sequence data yielding robust species resolution as demonstrated by both the tree topologies and the bootstrap values in Figs. 5 and 6. A comparable phylogenetic analysis using only the V4 and V5 regions of the 16S rRNA gene ( $400 \mathrm{bp})$ did not provide robust species node resolution (Additional file 1: Figures S14-S15).

Although MinION sequencing of individual DNA molecules represents a major advance in characterizing entire operons and does not require in silico assembly, it should be noted that the Nanopore R5-R7 error rates ( 15\%; [23]) are often higher than the error rates for other sequencing systems, such as PacBio (25-160x higher; [24]). To date, nanopore sequencing has mostly been used to re-sequence known genomes for testing the ability to provide long reads and improve error correction. For example, there are reports of complete genomes being assembled using only R7 Nanopore sequence data with accuracies of $99.5 \%$ for Escherichia coli K12 [25] and $99.8 \%$ for Francisella strains [24]. Additionally, a combination of nanopore reads recruiting Illumina short reads to create a synthetic consensus for assembly/alignment ( $\mathrm{NaS}$ fragments up to $60 \mathrm{~kb}$ in length) has been described with 99.99\% accuracy for Acinetobacter baylyi [26]. However, it is conceivable that the higher MinION error rate could overestimate the number of OTUs that are detected, as has been reported for the V3-V5 regions of the 16S rRNA genes using MiSeq approaches [27]. Using the shorter MiSeq reads, the OTUs in a model bacterial community were overestimated by a factor of 1.1-9.6x depending on the variable region and chimera removal using UCHIME. It is possible that the large number of singleton OTUs detected by the MinION reflect a higher sequence error. Interestingly, analysis of

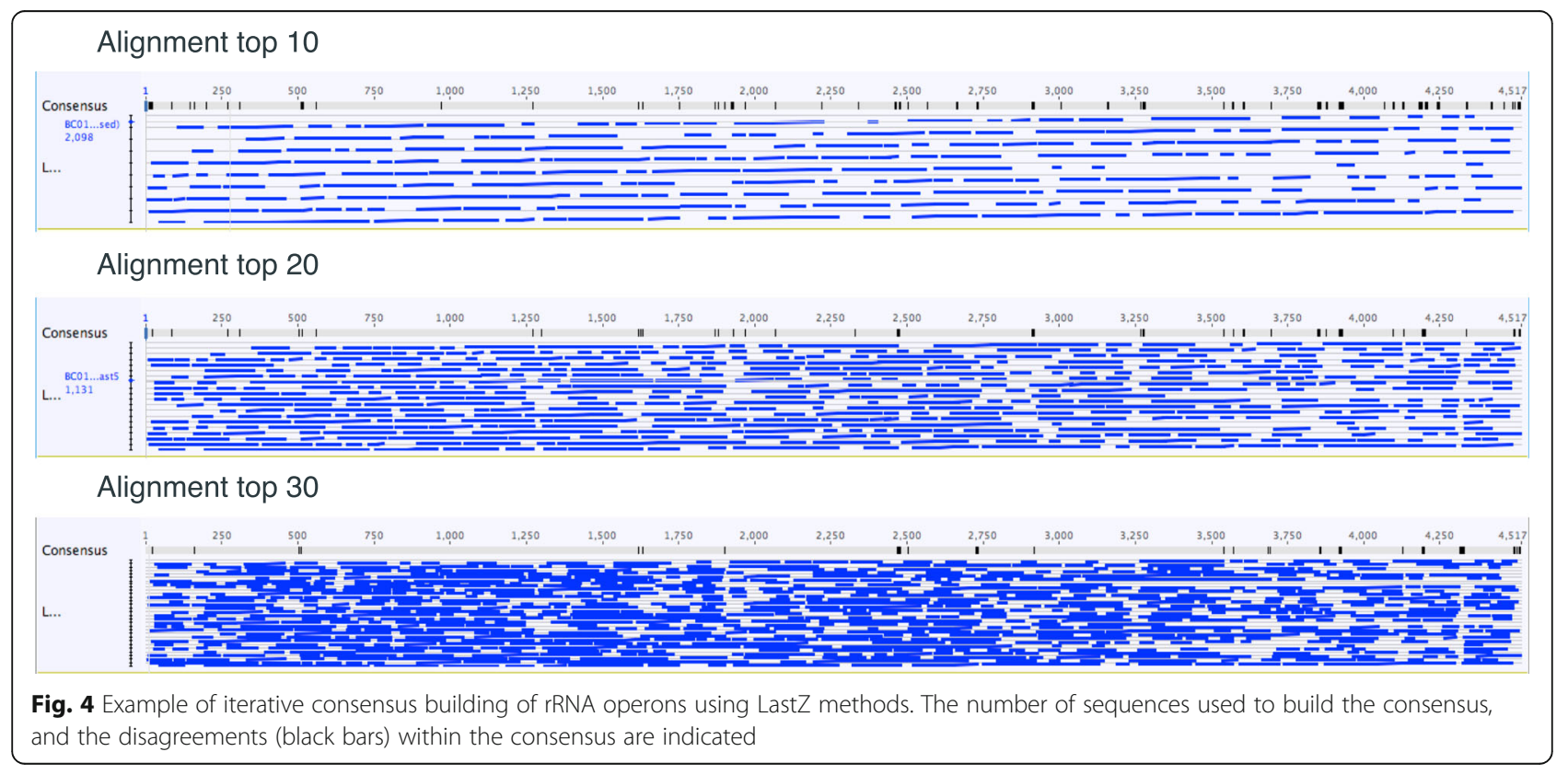




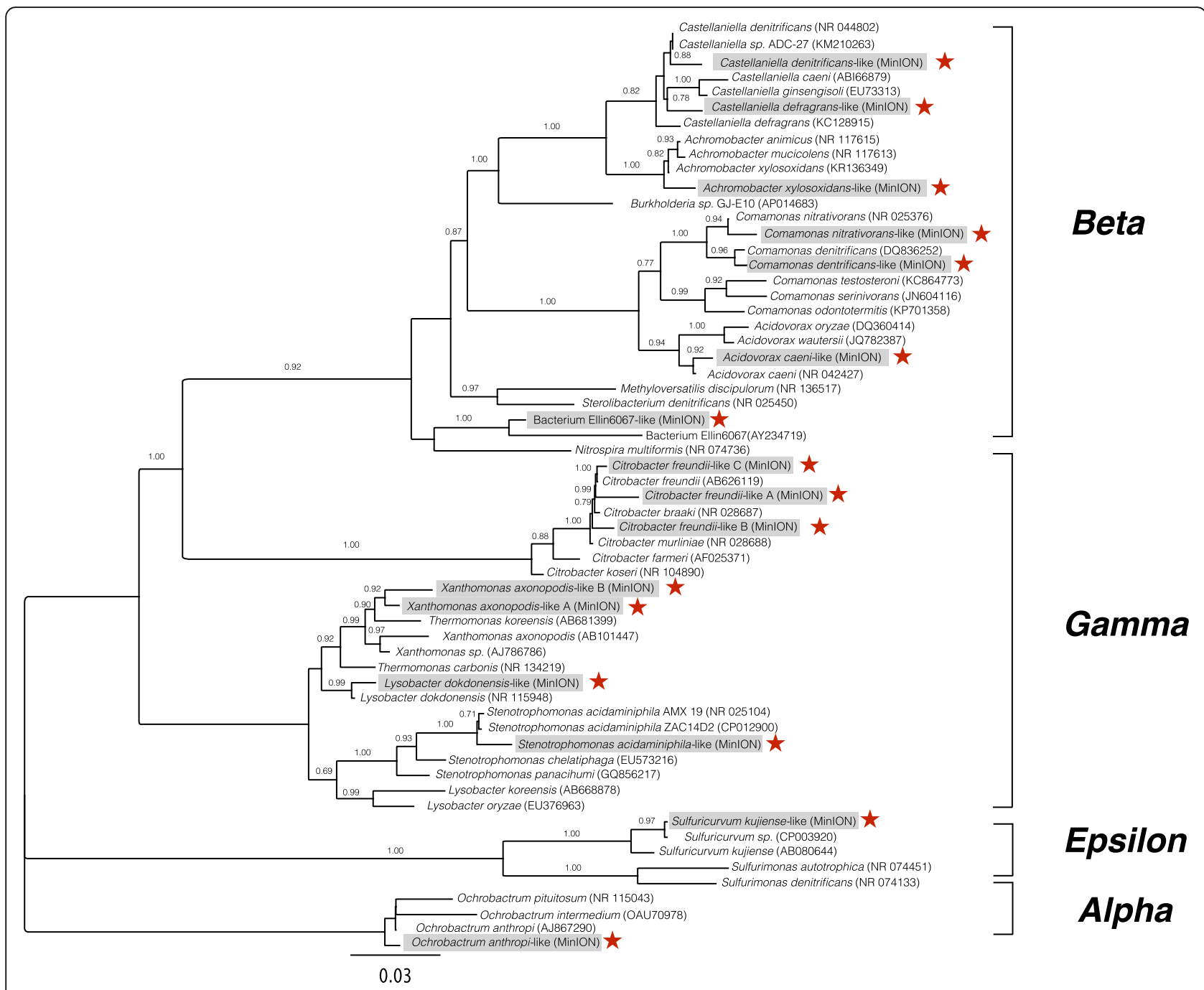

Fig. 5 Phylogenetic tree reconstruction for the Proteobacteria 165 rRNA genes from farm soil, a gray water bioreactor, and closely related matching reference sequences using FastTree for 1292 unambiguously aligned bases. The MinION sequences are indicated by boxes and stars

our DMegaBLAST results from the singleton MinION reads ( $>40 \%$ query coverage) indicated that the average identity was $81 \pm 5 \%$ over $1170 \pm 150 \mathrm{bp}$ for the $16 \mathrm{~S}$ rRNA gene $(n=2409)$. Based on the sensitivity analysis (Additional file 1: Figure S5), the singleton DMegaBLAST results suggest that many of our rare reads are potentially being correctly assigned to an OTU. Furthermore, ONT has recently released R9 flow cells and chemistry for the MinION with improved throughput and lower error rates which promise to enable more accurate OTU assignment and a much greater number of near complete rRNA operons to be assembled from other complex environments.

Finally, other researchers have begun using the MinION to determine near a full-length sequence of $16 \mathrm{~S}$ rRNA gene amplicons. Most of these studies have also tested model communities to demonstrate proof of concept. Specifically, researchers have tested E. coli K12 [28], a 3-member bacterial system containing Streptococcus and
Parvimonas [29], a 20-member model community using representative DNA from different bacterial phyla (e.g., Proteobacteria, Firmicutes, Bacteroides, Deinococcus, Actinobacteria) [30, 31]. All studies found that the MinION could provide a nearly full-length sequence of $16 \mathrm{~S}$ rRNA gene amplicons with accuracies ranging from 80 to $94 \%$ and could often obtain species-level resolution. Only a single study has utilized a complex "environmental"-type sample (mouse fecal material) [32] comparing Illumina and ONT Nanopore sequencing). While Shin et al. (2016) found nearly 1000 OTUs by Illumina methods, and they did not report the number of OTUs in their MinION data. However, they described the identification of more bacterial species using the nanopore data compared with the MiSeq $(n=16)$, and the authors could demonstrate robust phylogenetic resolution of species of Bifidobacterium and Bacteroides. In contrast with these prior studies, we used purified DNA from complex environment settings (soils 


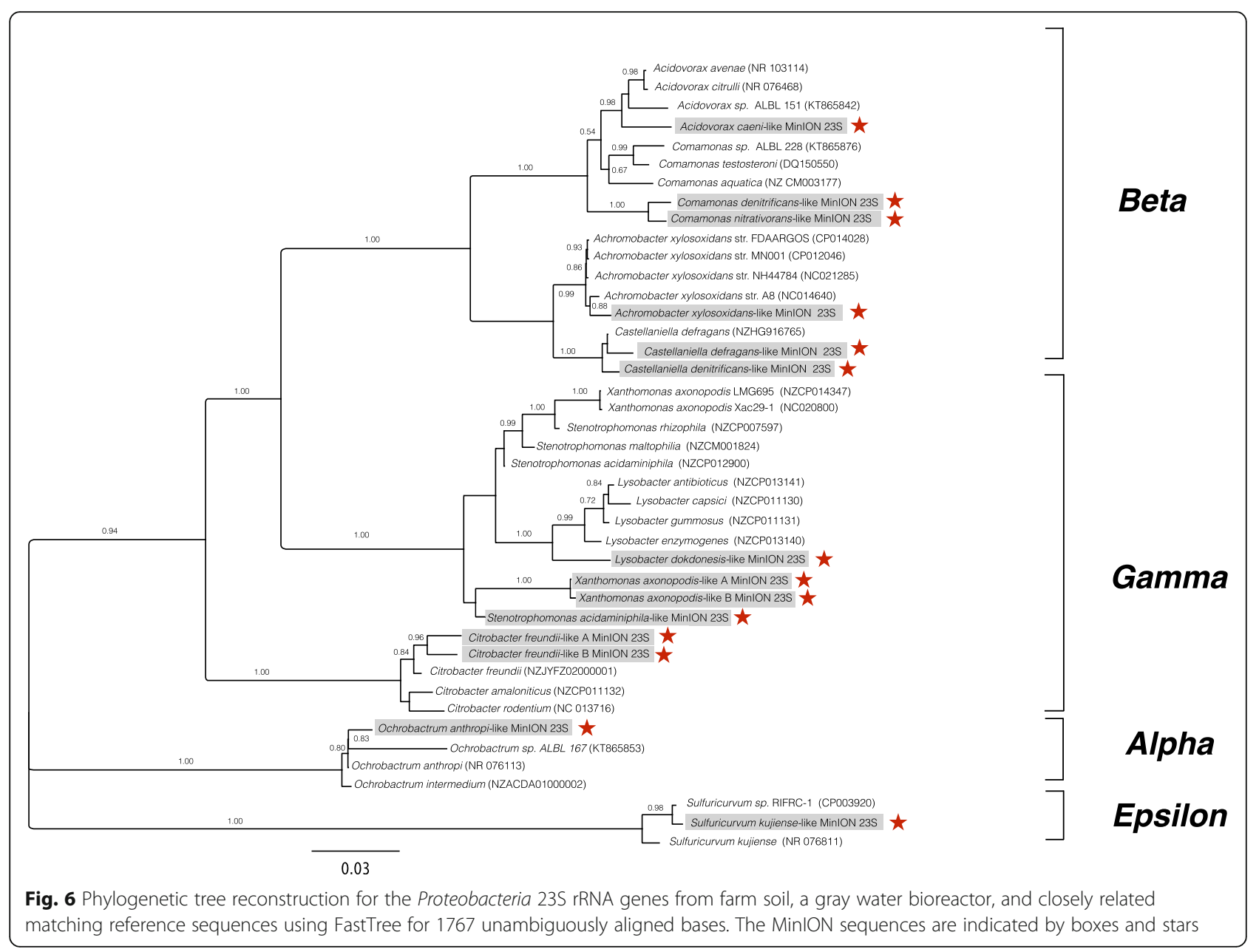

and bioreactors), containing a large number of unknown bacterial species and grouped the various MinION sequences by OTU to remove sequencing errors using an iterative consensus building approach.

\section{Conclusions}

Our analysis demonstrated that the MinION has the ability to provide rRNA operon sequence data of sufficient quality for characterizing the microbiota of complex environmental samples and provided results that are reproducible, quantitative, and consistent. Over 1000 OTUs could be detected from our test environmental sample mixture. However, further analysis of the errors in rare reads may be necessary to ensure accurate OTU assignment. The long-read capability of MinION allowed for robust bacterial species and strain resolution combining both $16 \mathrm{~S}$ and $23 \mathrm{~S}$ rRNA genes, consistent with previous reports $[30,32]$. Additionally, improvements in chemistry and library prep have led to increasing accuracy from 66 to $92 \%$ within the last few years [33] and ONT has released a newer version of their analysis software (MinKnow v51.3) that allows for local base calling on the host computer, rather than in the cloud using Metrichor. Given the MinION's low cost, small size, improving chemistry, and ability to analyze the nucleic acid data in real time, genetic analysis on mobile, and robotic platforms becomes feasible, as connectivity to the web is no longer required to analyze a sequence run.

\section{Methods \\ DNA extraction}

DNA from Rutgers farm soil and bioreactor samples [12] were extracted in triplicate (twice) using a modified CTAB extraction method [34]. Briefly, samples were amended with $100 \mu$ l of solution 1 (50 mM glucose, $10 \mathrm{mM}$ EDTA, $25 \mathrm{mM}$ Tris-Cl; pH 8.0) and subjected to five quick freeze/thaw cycles between liquid nitrogen temperatures and a $55{ }^{\circ} \mathrm{C}$ water bath. After these freeze/ thaw cycles, $450 \mu \mathrm{L}$ CTAB solution (4\% CTAB, $100 \mathrm{mM}$ Tris [pH 8.2], $20 \mathrm{mM}$ EDTA, $1.4 \mathrm{M} \mathrm{NaCl}), 0.14 \mathrm{M} \beta$ mercaptoethanol was added. The samples were quickly extracted $2 \mathrm{x}$ with $800 \mu \mathrm{l}$ phenol: chloroform: isoamyl alcohol; 25:24:1 (> pH 7.0). The aqueous phase of each extract was ethanol precipitated with the addition of $2 \mu \mathrm{g}$ 
of glycogen. The triplicate extracts were combined into a single stock for the farm soil and bioreactor to create end-member DNAs of very different microbial communities for this study. These end-member DNAs were brought to the same concentration and combined in different ratios, respectively: 0/100, 10/90, 20/80, 50/50, $75 / 25$, and $100 / 0$ (farm soil DNA/bioreactor DNA) for MinION sequencing (Fig. 1). Further DNA purification was done by combining $20 \mu \mathrm{l}$ of DNA, $20 \mu \mathrm{l}$ of $6 \mathrm{M} \mathrm{NaI}$, and $20 \mu \mathrm{l}$ of Ampure beads (Beckman Coulter; Brea, CA, USA). After DNA binding on a vortexer mixer for $10 \mathrm{~min}$, the beads were separated using a magnet and washed twice with freshly made $70 \%$ ethanol. DNA elution employed sterile water with a $55{ }^{\circ} \mathrm{C}$ treatment for $10 \mathrm{~min}$.

\section{Amplification of rRNA operons}

Bacterial ribosomal operons were amplified using modified 16S rRNA-27Forward primer $\left(5^{\prime}\right.$ TTT CTG TTG GTG CTG ATA TTG C-[barcode overhang for PCR labeling]-AGA GTT TGA TCC TGG CTC AG 3') [35] and modified 23S rRNA-2241Reverse primer (5' ACT TGC CTG TCG CTC TAT CTT C-[barcode overhang for PCR labeling]-ACC GCC CCA GTH AAA CT 3') [36]. Ribosomal operon amplicons were generated using AMPure bead purified DNA as follows: $10 \mathrm{ng}$ template DNA was combined with dNTP's, five units of Universe High-Fidelity Hot Start DNA polymerase (Biomake LLC, Houston, TX, USA), primers, and PCR buffer. Amplification conditions were $5 \mathrm{~min}$ at $94{ }^{\circ} \mathrm{C}$, followed by 27 cycles at $94{ }^{\circ} \mathrm{C}$ for $0.5 \mathrm{~min}$ and $72{ }^{\circ} \mathrm{C}$ for $1.5 \mathrm{~min}$. At 18 cycles, $10 \mu \mathrm{l}$ of amplification mixture was removed and stored at $-80{ }^{\circ} \mathrm{C}$. The amplification was allowed to proceed until 27 cycles and the product was visualized by agarose gel electrophoresis. Once clean, PCR product was observed, the 18 cycle mixture was cleaned with AMPure beads by bringing the volume up to $50 \mu \mathrm{l}$ with water, adding $50 \mu \mathrm{l}$ of $5 \mathrm{M} \mathrm{NaCl}, 50 \mu \mathrm{l}$ of $30 \%$ PEG/1.5 M NaCl, and $7 \mu \mathrm{l}$ of Ampure Beads. Ethanol washing and resuspension in $10 \mu \mathrm{l}$ of water were done as described above. Purified DNA $(1 \mu \mathrm{l})$ after 18 PCR cycles was added to a tube containing the ONT barcodes, and the amplification was repeated.

\section{Library preparation}

Library construction for the MinION relies on ligation of adaptor and hairpin to rRNA amplicons in order to perform nanopore sequencing. For this study, $100 \mathrm{ng}$ of each barcoded amplicon were combined (1200 ng total) into DNA Lo-Bind tubes at a volume of $85 \mu \mathrm{l}$ (by adding reagent grade PCR water) with $10 \mu \mathrm{l}$ end-repair buffer and $5 \mu \mathrm{l}$ of the end-repair enzyme (New England Biolabs, Ipswich, MA, USA). After a 20 min incubation at room temperature, the end-repair reaction was concentrated/purified by adding $100 \mu \mathrm{l}$ of $5 \mathrm{M} \mathrm{NaCl}$, $100 \mu \mathrm{l}$ of $30 \%$ PEG/1.5 M NaCl, and $15 \mu \mathrm{l}$ of Ampure Beads and allowed to bind for $10 \mathrm{~min}$ on a vortex shaker. The beads were removed from the supernatant using a magnet and washed twice with freshly made $70 \%$ ethanol. The end-repaired DNA was eluted in $25 \mu \mathrm{l}$ of water at $55{ }^{\circ} \mathrm{C}$ for $10 \mathrm{~min}$ and $\mathrm{dA}$-tailing was done by adding $3 \mu \mathrm{l}$ of tailing buffer and $2 \mu \mathrm{l}$ of enzyme (NEB) and incubating at $37^{\circ} \mathrm{C}$ for $10 \mathrm{~min}$. The DNA was repurified on AMPure beads using the $\mathrm{NaCl} / \mathrm{PEG}$ protocol as above and re-suspended in $15 \mu \mathrm{l}$ of water at $55{ }^{\circ} \mathrm{C}$ for $10 \mathrm{~min}$.

For the ligation, "half-reactions" were utilized with slight modifications. Fifteen microliters of DNA was combined with $9 \mu \mathrm{l}$ of water, $5 \mu \mathrm{l}$ of ONT adaptor mix, $1 \mu \mathrm{l}$ of HP adaptor, and $25 \mu \mathrm{l}$ of Blunt/TA ligase master mix (NEB). Additionally, a critical modification was to add 1-2 $\mu$ l of freshly prepared ATP solution $(\sim 4 \mathrm{mg} / \mathrm{ml})$. The mixture was incubated for $10 \mathrm{~min}$ at room temperature, then $0.5 \mu \mathrm{l}$ of HP tether was added, and the reaction was allowed to incubate an additional $10 \mathrm{~min}$. The library was then purified using streptavidin $\mathrm{C} 1$ magnetic beads as per ONT instructions with the exception that the elution was done by incubating the bead in $25 \mu \mathrm{l}$ elution buffer overnight at $4{ }^{\circ} \mathrm{C}$ then by a $30-$ min incubation at $37^{\circ} \mathrm{C}$. The library was loaded into R7 flow cells and run as per the manufacturer's instructions.

\section{QA/QC on Geneious}

After sequencing on the MinION, the 2D reads were opened using Poretools [37] and the corresponding fastA files were exported. These sequence files were subjected to QA/QC analysis by annotating in Geneious using six pairs each of universal 16S rRNA primer sequences (27F, 343F, 518F, 907F, 1392F, and 1492F) [35] and $23 \mathrm{~S}$ rRNA primer sequences (129F, 473F, 820F, 1623F, 2069F, and 2758F) [35, 36]. Only those files between 4 and $5 \mathrm{~kb}$ and containing at least two rRNA priming sites were retained for further analysis $(\sim 85 \%$ of the $2 \mathrm{D}$ sequences). These files were oriented in a uniform direction, and the 16S rRNA sequences were extracted in Geneious (Additional file 1: Figure S16).

\section{OTU determination}

The MinION 16S rRNA genes for each barcode were screened against an NCBI 16S rRNA gene bacterial and archaeal database (Bioproject 33175) using Discontinuous MegaBLAST in Geneious 10.1.2. Settings included a word size of 11 , gap cost of $5 / 2$, scoring of $2 /-3$, and a seed length of 18. The top BLAST output was exported as .csv files and opened in a spreadsheet program (e.g., Numbers, Excel) to group by best BLAST hit, count the number of OTUs, and parse for comparisons across samples. Additionally, the MinION sequences were 
analyzed by SINA online at the Arb-SILVA website (https://www.arb-silva.de/aligner). Settings included rejecting sequences $<70 \%$ identity, search-kmer candidates 100, lca-quorum 0.8, search-kmer length 10 using the SILVA, RDP, and Greengenes RefNR databases.

\section{Consensus reconstruction}

Thirty sequences representing a single OTU with the same top DMegaBLAST scores were copied into a separate folder and used to build a consensus rRNA operon from the host organism via an iterative LastZ alignment approach [22]. Initially, ten sequences were selected to build a consensus sequence by MUSCLE alignment using Geneious. This consensus was exported as text and imported in Pages to remove gaps. This MUSCLE consensus was used to re-align the original 10 operon sequences into a new LastZ consensus (termed "con 1A"). "Con 1A" was then used to align 20 of the rRNA operon sequences with LastZ to create "con $2 \mathrm{~A}$ ". "Con $2 \mathrm{~A}$ " was then used to align 30 of the rRNA operons to create a final consensus. The process was repeated with the next set of operon sequences (e.g., con 1B) and with the final set of 10 sequences (con 1C, etc.) All three final consensus sequences (A, B, C) were assessed for coverage and sequence length to choose a final consensus that best represents the OTU (Fig. 4). This final rRNA consensus sequence was annotated by selecting regions excluding the priming sites and screened by BLAST against the NR database to determine the full extent of the $16 \mathrm{~S}$ and $23 \mathrm{~S}$ rRNA genes.

\section{Phylogenetic tree analysis}

A maximum likelihood method (FastTree 2.1.5 with default settings) was used to reconstruct phylogenetic trees by first aligning full-length sequence for the ribosomal subunits with MUSCLE. The alignment was edited in Geneious to retain only unambiguously aligned bases (16S rRNA genes-1292 bp and 23S rRNA genes$1767 \mathrm{bp})$.

\section{Additional file}

Additional file 1: Figure S1. Comparison of the QIIME data analysis pipeline with this study of MinION data. Figure S2. Example of sequencing errors from individual MinION reads. Figure S3. Schematic diagram indicating the PCR primer sites for rRNA operon amplification and an agarose gel demonstrating amplicons. Figure S4. Percent of OTU classification results for the MinION reads by database using Discontinuous MegaBLAST (NCBI) or the ARB/SINA website (https:www.arb-silva.de/aligner; Greengenes, RDP, and SILVA). Figure S5. Test sequences used for assessing Discontinuous MegaBLAST for assigning operational taxonomic units (OTUs) to individual MinION reads. Figure S6. The number of barcoded reads using equal masses of amplicons for library creation. Figure S7. Average linear correlation coefficients for less abundant OTUs (with 3 or more data points) not shown in Fig. 3. Figure S8. Examples of 10 re-constructed rRNA operons from the MinlON. Figure S9. Comparison of 165 rRNA gene consensus sequences from reads in the 4 biological replicates. Figure S10.
Phylogenetic tree re-construction for the Actinobacteria 165 rRNA genes. Figure S11. Phylogenetic tree re-construction for the Actinobacteria $23 \mathrm{~S}$ rRNA genes. Figure S12. Phylogenetic tree re-construction for $16 \mathrm{~S}$ rRNA genes from other bacterial phyla. Figure S13. Phylogenetic tree reconstruction for $23 \mathrm{~S}$ rRNA genes from other bacterial phyla. Figure S14. Phylogenetic tree re-construction for the Proteobacteria using FastTree for 400 unambiguously aligned bases of the V4-V5 regions from the $16 \mathrm{~S}$ rRNA gene. Figure S15. Phylogenetic tree re-construction for the Proteobacteria using FastTree for 1292 unambiguously aligned bases from the nearly complete $16 \mathrm{~S}$ rRNA gene. Figure S16. Example of QA/ QC screening of the MinlON raw data by Geneious. Table S1. MinION read statistics. Table S2. Results of screening the "mutated" copies of three 16S rRNA genes using Discontinuous MegaBLAST as described in the text. (PDF $3614 \mathrm{~kb}$ )

\section{Acknowledgements}

The authors wish to thank Oxford Nanopore Technologies for creating the MinlON Access Program and supporting the Nanopore Community. Particular thanks to James Breyer and Andy Davies for their interest in our research projects and Concetta Dipace and Mike Micorescu for their helpful suggestions for LastZ data analysis.

\section{Funding}

This research was primarily funded by the National Science Foundation through an Ocean Technology and Interdisciplinary Program grant to LK (NSF \#1131022).

\section{Availability of data and materials}

All data is currently being made available at NCBI SRA (BioProject \#PRJNA383904).

\section{Authors' contributions}

LJK conceived and designed the experiments. KPD, LRM, and LJK performed the experiments. LJK and LRM designed the Apple Scripts. LK analyzed the data. $L K, L R M$, and $M M H$ discussed the findings and interpreted the results. LJK, KPD, LRM, and MMH wrote the paper. All authors read and approved the final manuscript.

\section{Ethics approval and consent to participate}

Not applicable.

\section{Competing interests}

The authors declare that they have no competing interests.

\section{Publisher's Note}

Springer Nature remains neutral with regard to jurisdictional claims in published maps and institutional affiliations.

\section{Author details}

'Department of Marine and Coastal Sciences, Rutgers University, 71 Dudley Rd, New Brunswick, NJ 08901-8521, USA. ²Department of Environmental Sciences, Rutgers University, New Brunswick, NJ 08901, USA. ${ }^{3}$ Department of Biochemistry and Microbiology, Rutgers University, New Brunswick, NJ 08901, USA.

Received: 25 April 2017 Accepted: 30 August 2017

Published online: 15 September 2017

\section{References}

1. Stahl DA, Lane DJ, Olsen GJ, Pace NR. Characterization of a Yellowstone hot spring microbial community by $5 \mathrm{~S}$ rRNA sequences. Appl Environ Microbiol. 1985:49:1379-84.

2. Olsen GJ, Lane DJ, Giovannoni SJ, Pace NR, Stahl DA. Microbial ecology and evolution: a ribosomal RNA approach. Ann Rev Microbiol. 1986;40:337-65.

3. Muyzer G, De Waal EC, Uitterlinden AG. Profiling of complex microbial populations by denaturing gradient gel electrophoresis analysis of polymerase chain reaction-amplified genes coding for 165 rRNA. Appl Environ Microbiol. 1993;59:695-700.

4. Avaniss-Aghajani E, Jones K, Chapman D, Brunk C. A molecular technique for identification of bacteria using small subunit ribosomal RNA sequences. BioTechniques. 1994;17:144-6. 
5. Widjojoatmodljo MN, Fluit ADC, Verhoer J. Molecular identification of bacteria by fluorescence-based PCR-single-strand conformation polymorphism analysis of the 16S rRNA gene. J Clin Microbiol. 1995;33:2601-6.

6. Sogin ML, Morrison HG, Huber JA, Welch DM, Huse SM, Neal PR, Arrieta JM, Herndl GJ. Microbial diversity in the deep sea and the underexplored "rare biosphere". Proc Natl Acad Sciences. 2006;103:12115-20.

7. Roesch LFW, Fulthorpe RR, Riva A, Casella G, Hadwin AKM, Kent AD, Daroub $\mathrm{SH}$, Camargo FAO, Farmerie WG, Triplett EW. Pyrosequencing enumerates and contrasts soil microbial diversity. ISME J. 2007;1:283-90.

8. Lazarevic V, Whiteson K, Huse S, Hernandez D, Farinelli L, Østerås M, Schrenzel J, François P. Metagenomic study of the oral microbiota by Illumina high-throughput sequencing. J Microbiol Meth. 2009;79:266-71.

9. Whiteley AS, Jenkins S, Waite I, Kresoje N, Payne H, Mullan B, Allcock R, O'Donnell A. Microbial $16 \mathrm{~S}$ rRNA ion tag and community metagenome sequencing using the ion torrent (PGM) platform. J Microbiol Meth. 2012;91:80-8.

10. Schneider GF, Dekker C. DNA sequencing with nanopores. Nature Biotech. 2012;30:326-8.

11. Wang Y, Yang Q, Wang Z. The evolution of nanopore sequencing. Front Gen. 2015;449:1-20. doi:10.3389/fgene.2014.00449.

12. McGuinness LM, Salganik M, Vega L, Pickering KD, Kerkhof LJ. Replicability of bacterial communities in denitrifying bioreactors as measured by $P C R / T$ RFLP analysis. Env Science and Tech. 2006;40:509-15.

13. Jiang $X T$, Peng $X$, Deng $G H$, Sheng HF, Wang $Y$, Zhou HW, Tam NFY. Illumina sequencing of $16 \mathrm{~S}$ rRNA tag revealed spatial variations of bacterial communities in a mangrove wetland. Microb Ecol. 2013;66:96-104.

14. Hong C, Si Y, Xing Y, Li Y. Illumina MiSeq sequencing investigation on the contrasting soil bacterial community structures in different iron mining areas. Environ Sci Pollut Res. 2015;22:10788-99.

15. Wu X, Zhang H, Chen J, Shang S, Wei Q, Yan J, Tu X. Comparison of the fecal microbiota of dholes high-throughput Illumina sequencing of the V3-V4 region of the 16S rRNA gene. Appl Microbiol Biotechnol. 2016;100:3577-86.

16. Li LT, Yan BL, Li SH, Xu JT, An XH. A comparison of bacterial community structure in seawater pond with shrimp, crab, and shellfish cultures and in non-cultured pond in Ganyu. Eastern China Ann Microbiol. 2016;66:317-28.

17. Ye L, Shao MF, Zhang T, Tong AHY, Lok S. Analysis of the bacterial community in a laboratory-scale nitrification reactor and a wastewater treatment plant by 454-pyrosequencing. Water Res. 2011:45:4390-8.

18. Hand D, Wallis C, Colyer A, Penn CW. Pyrosequencing the canine faecal microbiota: breadth and depth of biodiversity. PLoS One. 2013;8:e53115.

19. Wang A, Yao Z, Zheng W, Zhang H. Bacterial communities in the gut and reproductive organs of Bactrocera minax (Diptera: Tephritidae) based on 454 pyrosequencing. PLoS One. 2014;9:e106988.

20. Myer PR, MS FHC, TPL S. Evaluation of 165 rRNA amplicon sequencing using two next-generation sequencing technologies for phylogenetic analysis of the rumen bacterial community in steers. J Microbiol Meth. 2016;127:132-40.

21. CLC I, Loose M, Tysone JR, de Cesare M, Brown BL, Jain M, Leggett RM, Eccles DA, Zalunin V, Urban JM, Piazza P, Bowden RJ, Paten B, Mwaigwisya S, Batty EM, Simpson JT, Snutch TP, Birney E, Buck D, Jansen HJ GS, O'Grady J, Olsen HE. MinION analysis and reference consortium: phase 1 data release and analysis. F1000Research. 2015;4:1075.

22. Harris RS Improved pairwise alignment of genomic DNA. PhD thesis. The Center for Comparative Genomics and Bioinformatics, The Pennsylvania State University, 501 Wartik Laboratory, University Park, PA 16802; 2007.

23. Loman NJ, Watson M. Successful tSest launch for nanopore sequencing. Nat Methods. 2015:12:303-4

24. Karlsson E, Lärkeryd A, Sjödin A, Forsman M, Stenberg P. Scaffolding of a bacterial genome using MinION nanopore sequencing. Nature Scientific Reports. 2015; doi:10.1038/srep11996.

25. Loman NJ, Quick J, Simpson JT. A complete bacterial genome assembled de novo using only nanopore sequencing data. Nature Meth. 2015; doi:10.1038/nMeth.3444.

26. Madoui M-A, Engelen S, Cruaud C, Belser C, Bertrand L, Alberti A, Lemainque A, Wincker P, Aury J-M. Genome assembly using Nanoporeguided long and error-free DNA reads. BMC Genomics. 2015;16:327. doi:10.1186/s12864-015-1519-z

27. Kozich JJ, Westcott SL, Baxter NT, Highlander SK, Schloss PD. Development of a dual-index sequencing strategy and curation pipeline for analyzing amplicon sequence data on the MiSeq Illumina sequencing platform. Appl Environ Microbiol. 2013;79:5112-20.

28. Kilianski A, Haas JL, Corriveau EJ, Liem AT, Willis KL, Kadavy DR, Rosenzweig CN, Minot SS. Bacterial and Viral identification and differentiation by amplicon sequencing on the MinION nanopore sequencer. GigaScience. 2015:4:12; doi:10.1186/s13742-015-0051-z.

29. Li C, Chang KR, Hui Boey EJ, Qi Ng AH, Wilm A, Nagarajan N. INC-Seq: accurate single molecule reads using nanopore sequencing. GigaScience. 2016:5:34. doi:10.1186/s13742-016-0140-7.

30. Benitez-Paez A, Portune KJ, Sanz Y. Species-level resolution of 165 rRNA gene amplicons sequenced through the MinION portable nanopore sequencer. GigaScience. 2016;5:4. doi:10.1186/s13742-016-0111-z.

31. Brown BL, Watson M, Minot SS, Rivera MC, Franklin RB. MinIONTM nanopore sequencing of environmental metagenomes: a synthetic approach. GigaScience. 2017:6:1-10.

32. Shin J, Lee S, Go M-J, Lee SY, Kim SC, Lee C-H, Cho B-K. Analysis of the mouse gut microbiome using full-length 16S rRNA amplicon sequencing. Nature Sci Rep. 2016;6:29681. doi:10.1038/srep29681.

33. Jain M, Olsen HE, Paten B, Akeson M. The Oxford Nanopore MinlON: delivery of nanopore sequencing to the genomics community. Genome Biol. 2016;17:239. doi:10.1186/s13059-016-1103-0.

34. Männistö MK, Tiirola M, Häggblom MM. Effect of freeze-thaw cycles on bacterial communities of arctic tundra soil. Microb Ecol. 2009;58:621-31.

35. Lane, D. J. 1991. 16S/23S rRNA sequencing. In: Stackebrandt E and Goodfellow M, editors. Nucleic acid techniques in bacterial systematics. Chichester, England: John Wiley \& Sons Ltd.; 1991. p. 115-175.

36. Hunt DE, Klepac-Ceraj V, Acinas SG, Gautier C, Bertilsson S, Polz MF. Evaluation of 23S rRNA PCR primers for use in phylogenetic studies of bacterial diversity. Appl Environ Microbiol. 2006;72:2221-5.

37. Loman NJ, Quinlan AR. Poretools: a toolkit for analyzing nanopore sequence data. Bioinformatics. 2014;30:3399-401.

\section{Submit your next manuscript to BioMed Central and we will help you at every step:}

- We accept pre-submission inquiries

- Our selector tool helps you to find the most relevant journal

- We provide round the clock customer support

- Convenient online submission

- Thorough peer review

- Inclusion in PubMed and all major indexing services

- Maximum visibility for your research

Submit your manuscript at www.biomedcentral.com/submit
) Biomed Central 